Research Article

\title{
Rate and Spraying Frequency Determination of Propiconazole Fungicide for the Management of Garlic Rust at Woreilu District, Northeastern Ethiopia
}

\author{
Admasie Kassaw (D, Aderajew Mihretie, and Abebe Ayalew \\ Sirinka Agricultural Research Centre, P. O. Box 74, Woldia, Ethiopia \\ Correspondence should be addressed to Admasie Kassaw; admasiek70@gmail.com
}

Received 13 August 2020; Revised 26 January 2021; Accepted 8 February 2021; Published 25 February 2021

Academic Editor: Gábor Kocsy

Copyright (c) 2021 Admasie Kassaw et al. This is an open access article distributed under the Creative Commons Attribution License, which permits unrestricted use, distribution, and reproduction in any medium, provided the original work is properly cited.

\begin{abstract}
Rust is a major disease of garlic in Ethiopia. A field experiment was conducted at Kabe and Segno Kebeles of Woreilu District in 2019 to determine the appropriate rate and spraying frequency of propiconazole fungicide and select cost-effective management options of garlic rust. A randomized complete block design with three replications was used. The combination of five rates and three spraying frequencies of propiconazole fungicide and unsprayed treatments was evaluated. A combination of rate and spraying frequencies of propiconazole significantly reduced disease with correspondingly increased bulb yield of garlic. The minimum percentage severity index and area under disease progress curve were scored from plots treated by all rates with three times spraying frequency of Tilt fungicide application. The highest bulb yield was obtained from plots treated with all fungicide rates with three times spraying frequencies of propiconazole application. But the highest (6896.36\%) marginal rate of return was obtained from plots treated with $0.5 \mathrm{~L}$ with two times spraying. Generally, 0.5 liters with two times spraying of propiconazole was effective in controlling garlic disease and get the highest cost-benefit advantage as compared with other treatments. Therefore, it could be recommended for the management of garlic rust in Ethiopia.
\end{abstract}

\section{Introduction}

Garlic (Allium sativum L.) belongs to the Alliaceae family, and it is an important edible bulbous crop [1]. The origin of garlic is assumed to be in Central Asia and has spread to other countries through trade and colonization [2]. Garlic has been used in China and India for more than 5000 years, and in Egypt since 2000 BC [3]. It is the foremost important crop and ranks second next to onion within the world [4]. Garlic is one of the main vegetable crops in the world and used as a seasoning in many foods throughout the globe. Also, it has medicinal value which is well known within the control and treatment of hypertension, worms, germs, bacterial and fungal diseases, diabetes, cancer, ulcer, rheumatism, etc. [5]. Garlic is one of the most important vegetable crops in Ethiopia and is used as a component of food and has also a tremendous use in the formulation of local medicines.
The production and productivity of garlic are low in many parts of the world including in Ethiopia due to biotic and abiotic factors affecting its yield and yield-related traits. Fungal diseases are major problems for production, productivity, and as well as the quality of the crop [6]. For example, garlic rust and bulb rot diseases are the major limiting factors regarding production and productivity of garlic [7].

Garlic rust (Puccinia allii) is the most important and serious disease of garlic in many countries of the world including in Ethiopia [7-9]. The disease is one of the most significant constraints to garlic productions, causing up to $51 \%$ of bulb yield losses [8]. In Ethiopia, P. allii declines the productivity of garlic bulb yield and causes a $58.7 \%$ yield loss [9]. The maximum bulb weight loss from the unsprayed plots to that of the Tilt sprayed plot was 55.9\% [10].

Management of garlic rust through the application of fungicides could be an important practice to control the 
disease [7]. According to Admasie et al. [11], the application of propiconazole (Tilt) fungicide could minimize the severity of garlic rust and improve total bulb yield as compared with other fungicides and control plots. Rex Due and propiconazole fungicides were found superior in managing of garlic rust and gave the highest yield as compared with other treatments [10]. The use of fungicides in controlling garlic rust improved the production and productivity of garlic bulb yield $[9,12,13]$.

The use of propiconazole fungicide for managing of garlic rust has been the most studied aspect of rust management in Ethiopia [11-13]. In the study areas, Tilt 250EC fungicide was recommended for the management of garlic rust disease [11]. Spraying of propiconazole for the control of the disease at different rates and spraying frequency may have different effects on the development of the disease. In addition to this, there is limited information on rate and spraying frequency determination of propiconazole in the study area. Therefore, the present study was initiated to determine an appropriate rate and spraying frequency of propiconazole fungicide and to select cost-effective fungicide rates and frequency for the management of garlic rust.

\section{Materials and Methods}

2.1. Experimental Design and Materials. The field experiment was done during 2019 main cropping season at Woreilu District on two farms (Kabe and Segno Kebeles). The field experiment was arranged in a randomized complete block design (RCBD) with three replications. The experiment had 16 treatments (Table 1), with a combination of five rates of propiconazole $(0.5,0.75,1$, 1.25 , and $1.5 \mathrm{~L} / \mathrm{Ha}$ ) and three spraying frequencies (one, two, and three times spray) and unsprayed treatment as a control check. The first spray of fungicides was done at 72 days after planting and continued on the bases of treatment spraying frequency at 10-day interval. Fungicide applications was done by using hand sprayer for each plot. A plastic sheet was used during fungicide application to prevent fungicide drift or cross-contamination for garlic field trials. The plot size of the experiment was $2.4 \times 2.4 \mathrm{~m}$ with a plant spacing of $10 \mathrm{~cm}$ and $40 \mathrm{~cm}$ row spacing and $0.5 \mathrm{~m}$ and $1 \mathrm{~m}$ spacing between plots and experimental blocks, respectively. There were six rows per plot and the middle four rows with a net plot area of $3.84 \mathrm{~m}^{2}$ (excluding the two border rows) were used for data collection. A spacing of 0.5 and 1 meter was used to separate each plot and block, respectively.

The experiment was done using a local garlic variety as planting material. Uniform medium-sized garlic cloves were used as planting material for all treatments. All agronomic practices such as earthing-up, ridging, weeding, and cultivation were applied uniformly for all treatments in each plot.

2.2. Disease Data Collected. Disease incidence percentage was recorded by counting of plants showing visible symptoms of late blight in the central rows and the data were
TABLE 1: Treatment combination of five propiconazole rates and three spraying frequencies.

\begin{tabular}{lc}
\hline TRT & Treatment combination \\
\hline 1 & $0.5 * 1 \mathrm{X}$ \\
2 & $0.5 * 2 \mathrm{X}$ \\
3 & $0.5 * 3 \mathrm{X}$ \\
4 & $0.75 * 1 \mathrm{X}$ \\
5 & $0.75 * 2 \mathrm{X}$ \\
6 & $0.75 * 3 \mathrm{X}$ \\
7 & $1 * 1 \mathrm{X}$ \\
8 & $1 * 2 \mathrm{X}$ \\
9 & $1 * 3 \mathrm{X}$ \\
10 & $1.25 * 1 \mathrm{X}$ \\
11 & $1.25 * 2 \mathrm{X}$ \\
12 & $1.25 * 3 \mathrm{X}$ \\
13 & $1.5 * 1 \mathrm{X}$ \\
14 & $1.5 * 2 \mathrm{X}$ \\
15 & $1.5 * 3 \mathrm{X}$ \\
16 & Control \\
\hline
\end{tabular}

expressed as a percentage of the total assessed plants. The disease incidence was calculated by the following formula:

Disease incidence $=\frac{\text { number of diseased plants }}{\text { total number of plants inspected }} \times 100$.

Disease severity was recorded by visually estimating the percentage of leaf area diseased from 10 randomly taken and pretagged plants in the middle two rows of each plot. The severity was scored five times every 10 days starting from the onset of the disease by using a standard disease scale of 1-5 rust severity, where $1=1-10 \%, 2=11-25 \%, 3=26-50 \%$, $4=51-75 \%$, and $5=76-100 \%$ of the leaf surface covered with lesions [8], and average severity of the 10 plants per plot was used for statistical analysis. Each severity value was converted into percentage severity index (PSI) for analysis [14].

$$
\mathrm{PSI}=\frac{\mathrm{Snr}}{\mathrm{Npr} \times \mathrm{Msc}} \times 100,
$$

where Snr is the summation of numerical ratings, Npr is total number of plants rated, and Msc is the maximum score of the scale.

The area under disease progress curve (AUDPC) was calculated for each treatment from the assessment of disease severity using the following formula [15]:

$$
\mathrm{AUDPC}=\sum_{i=1}^{n-1} 0.5\left(\left(x_{i+1}+x_{i}\right)\left(\mathrm{t}_{i+1}-t_{i}\right)\right)
$$

where $x$ is disease severity at ith observation, $n$ is the total number of days disease assessed, and $t$ is the time at the ith observation (in days numbered sequentially beginning with the initial assessment).

2.3. Yield and Yield Component Data Collection. Yield and yield component data such as bulb diameter, bulb weight, number of cloves per bulb, clove weight, and bulb yield were recorded based on their standard measurements. 
2.4. Increase in Relative Yield Loss and Percentage Yield. Relative yield loss due to garlic rust was measured as percentage yield reduction of unsprayed plots compared with the most protected plot using the following formula of Robert and Janes [16]:

$$
\mathrm{RPYL}=\frac{\mathbf{Y P}-\mathbf{Y T}}{\mathbf{Y P}} \times 100,
$$

where RPYL is relative percent yield loss, YP is yield from the maximum protected plot, and YT is yield from plots of other treatments.

The percent yield increase (PYI) was calculated based on Lung'aho et al.'s [17] suggested formula:

PYI $=\frac{\text { bulb yield of a fungicide treated plot }- \text { yield of control plot }}{\text { yield of control plot }} \times 100$.

2.5. Cost-Benefit Assessment. Cost-benefit assessment of each treatment was analyzed partially, and the marginal rate of return was computed by considering the variable cost available in the respective treatment [2]. Variable costs included chemical and labor cost for chemical application. The price of propiconazole (Tilt 250EC) was Ethiopian Birr $1100 \mathrm{~L}^{-1}$ and the labor cost of Birr 44 man-days was used. At the end of the production, total gross benefit of the field was calculated from the bulb yield of garlic with the local market price (Birr $80 \mathrm{~kg}^{-1}$ ) of Woreilu District. Total input costs were obtained from the summation of total cost that varied and fixed cost of the production used. The cost and benefit of each treatment were analyzed partially and the marginal rate of return (MRR) was computed by considering the variable cost available in the respective treatment. Yield and economic data were computed to compare the advantage of rates and spraying of fungicide applications in different treatment combinations. The marginal rate of return provides the value of the benefit obtained per the amount of additional cost incurred percentage.

$$
\mathrm{MRR}=\frac{\mathrm{MNB}}{\mathrm{MC}},
$$

where MRR is marginal rate of returns, MNB is marginal net benefit compared with control, and MC is marginal cost compared with control.

2.6. Data Analysis. Data on disease incidence, disease severity, AUDPC, yield, and yield component parameters were subjected to analysis of variance (ANOVA) for each data by using GenStat version 18.0 Software (GenStat, 2015). By using Duncan's multiple range test, treatments mean separation was done. Correlation analysis was done to know the association of disease parameters with yield. The linear regression model was used to predict the relationship of AUDPC and bulb yield by using GenStat version 18.0 Software.

\section{Results and Discussion}

3.1. Disease Assessment. The initial indications of the rust disease were observed at 67 and 69 days after planting (DAP) at Kabe and Segno, respectively. However, garlic rust assessment was started in both locations at 72 days after planting (DAP) (Tables 1 and 2).

3.2. Disease Incidence. The incidence of the disease was analyzed only from the first assessment date (72DAP) because at the second assessment the incidence was 100\% in all the plots at Kabe location, whereas at Segno location the incidence of the disease was analyzed only from the first and second assessment dates because at the third assessment the disease showed in all the plots.

Propiconazole spray at a different rate and spraying frequency showed a significant difference $(p<0.05)$ on disease incidence at both locations (Tables 2 and 3). At Kabe, the lowest disease incidence (70\%) was scored from plots treated with $1.25 \mathrm{~L}$ rate in two times spraying frequency, whereas the highest disease (100\%) incidence was observed from plots treated with 0.75 rates at three times spray and at the rate of one liter with two times spraying frequency (Table 2). Similarly, at Segno the highest disease incidence $(100 \%)$ was recorded from the control plot and the lowest (70\%) was scored from plots sprayed 1.5-liter propiconazole with two times spraying frequency (Table 3).

3.3. Percentage Severity Index. The percentage severity index showed significance difference $(p<0.05)$ among treatments in all assessment dates except the initial assessment date (72DAP) at both locations (Tables 2 and 3). At the final disease assessment (112DAP), the highest PSI (79.33 and $78.67 \%$ ) was recorded from the control plots at Kabe and Segno locations, respectively. Also, the lowest (17.33\%) PSI was scored from plots treated with 1.5 liters with three times spraying frequencies at Kabe (Table 2) and 20.67\% scored from 1 liter with three times spray at Segno (Table 3). The finding of the result indicated three times the foliar sprays of Tilt at ten-day interval could be effective in reducing the severity of garlic rust at both locations. Moreover, three and two times spraying frequencies with all rates of Tilt fungicides were the best management options of garlic rust. But partial budget analysis indicated that minimum rate $\left(0.5 \mathrm{~L} \mathrm{ha}^{-1}\right)$ at two times spraying frequency of tilt fungicide was the most profitable compared to other combinations. Similarly, the lowest PSI of garlic rust was recorded at sevenday interval of natura fungicide sprayed plots at the rate of $0.75 \mathrm{~L} / \mathrm{ha}$ and $0.5 \mathrm{~L} / \mathrm{ha}$ [18]. According to Worku et al.'s [12] research result, garlic plots treated with propiconazole showed the lowest disease severity (3.72\%). All fungicidetreated plots gave significantly $(p<0.05)$ reduced disease severity compared to the untreated check plots [13].

3.4. Area under Disease Progress Curve (AUDPC). The analysis of variance revealed that statistically significant $(p<0.001)$ differences were shown among treatments on AUDPC at both Kabe and Segno locations (Tables 2 and 3). The highest AUDPC (1860 and 1853\%-days) was recorded from the control plots of Kabe and Segno locations, respectively. Similarly, the lowest (720\%-days) AUDPC was 
TABle 2: The mean of garlic rust disease incidence, PSI at d/t days, and AUDPC data at Kabe in 2019 cropping season.

\begin{tabular}{|c|c|c|c|c|c|c|c|}
\hline & Incidence & & & PSI & & & AUDPC \\
\hline Trt & 72DAP & 72DAP & 82DAP & 92DAP & 102DAP & 112DAP & $\%$ in days \\
\hline $0.5 * 1 \mathrm{X}$ & $96.67^{c}$ & 10 & $20.67^{\mathrm{a}}$ & $36.33^{\mathrm{d}}$ & $40.67^{\mathrm{e}}$ & $49.33^{\mathrm{d}}$ & $1273^{\mathrm{g}}$ \\
\hline $0.5 * 2 \mathrm{X}$ & $86.67^{\mathrm{abc}}$ & 7.33 & $20.67^{\mathrm{a}}$ & $35.33^{\mathrm{d}}$ & $37.33^{\mathrm{de}}$ & $34.67^{\mathrm{bcd}}$ & $1143^{\text {efg }}$ \\
\hline $0.5 * 3 \mathrm{X}$ & $96.67^{\mathrm{c}}$ & 9.33 & $18.67^{\mathrm{a}}$ & $30.67^{\mathrm{bcd}}$ & $32.67^{\text {cde }}$ & $25.67^{\mathrm{abc}}$ & $995^{\text {cde }}$ \\
\hline $0.75 * 1 \mathrm{X}$ & $83.33^{\mathrm{abc}}$ & 6.67 & $19.33^{\mathrm{a}}$ & $36.33^{\mathrm{d}}$ & $40^{\mathrm{e}}$ & $41.33^{\mathrm{cd}}$ & $1197^{\mathrm{fg}}$ \\
\hline $0.75 * 2 \mathrm{X}$ & $96.67^{\mathrm{c}}$ & 9.33 & $20.67^{\mathrm{a}}$ & $35.33^{\mathrm{cd}}$ & $33.33^{\text {cde }}$ & $32.67^{\mathrm{abc}}$ & $1103^{\mathrm{ef}}$ \\
\hline $0.75 * 3 \mathrm{X}$ & $100^{c}$ & 8.67 & $18^{\mathrm{a}}$ & $28.67^{\mathrm{abcd}}$ & $26.67^{\mathrm{abc}}$ & $25.33^{\mathrm{abc}}$ & $903^{\mathrm{bc}}$ \\
\hline $1 * 1 \mathrm{X}$ & $73.33^{\mathrm{ab}}$ & 4.67 & $19.33^{\mathrm{a}}$ & $26.33^{\mathrm{ab}}$ & $39.33^{\text {de }}$ & $40.67^{\mathrm{cd}}$ & $1077^{\text {def }}$ \\
\hline $1 * 2 \mathrm{X}$ & $100^{c}$ & 10 & $18^{\mathrm{a}}$ & $24^{\mathrm{ab}}$ & $30^{\mathrm{bcd}}$ & $32^{\mathrm{abc}}$ & $930^{\mathrm{bcd}}$ \\
\hline $1 * 3 \mathrm{X}$ & $83.33^{\mathrm{abc}}$ & 6.67 & $18^{\mathrm{a}}$ & $25.67^{\mathrm{ab}}$ & $24.67^{\mathrm{abc}}$ & $22^{\mathrm{ab}}$ & $827^{\mathrm{ab}}$ \\
\hline $1.25 * 1 \mathrm{X}$ & $83.33^{\mathrm{abc}}$ & 8.67 & $14^{\mathrm{a}}$ & $27.33^{\mathrm{abc}}$ & $36.67^{\text {de }}$ & $39.33^{\mathrm{cd}}$ & $1020^{\text {cde }}$ \\
\hline $1.25 * 2 \mathrm{X}$ & $70^{\mathrm{a}}$ & 4 & $15.33^{\mathrm{a}}$ & $22.67^{\mathrm{ab}}$ & $27.33 \mathrm{a}^{\mathrm{bc}}$ & $26^{\mathrm{abc}}$ & $803^{\mathrm{ab}}$ \\
\hline $1.25 * 3 \mathrm{X}$ & $83.33^{\mathrm{abc}}$ & 6.67 & $17.33^{\mathrm{a}}$ & $29.33^{\mathrm{abcd}}$ & $22^{\mathrm{ab}}$ & $19.33^{\mathrm{ab}}$ & $817^{\mathrm{ab}}$ \\
\hline $1.5 * 1 \mathrm{X}$ & $96.67^{\mathrm{c}}$ & 9.33 & $14.67^{\mathrm{a}}$ & $24.67^{\mathrm{ab}}$ & $22.67^{\mathrm{ab}}$ & $26.67^{\mathrm{abc}}$ & $800^{\mathrm{ab}}$ \\
\hline $1.5 * 2 \mathrm{X}$ & $93.33^{b c}$ & 8.67 & $18.67^{\mathrm{a}}$ & $23.33^{\mathrm{ab}}$ & $21.33^{\mathrm{ab}}$ & $18.67^{\mathrm{ab}}$ & $770^{\mathrm{ab}}$ \\
\hline $1.5 * 3 \mathrm{X}$ & $93.33^{b c}$ & 8.67 & $18^{\mathrm{a}}$ & $21.67^{\mathrm{a}}$ & $19.33^{\mathrm{a}}$ & $17.33^{\mathrm{a}}$ & $720^{\mathrm{a}}$ \\
\hline Control & $86.67^{\mathrm{abc}}$ & 7.33 & $30.67^{\mathrm{b}}$ & $48.67^{\mathrm{e}}$ & $63.33^{\mathrm{f}}$ & $79.33^{\mathrm{e}}$ & $1860^{\mathrm{h}}$ \\
\hline$\overline{G M}$ & 89 & 7.88 & 18.88 & 29.77 & 32.33 & 33.1 & 1015 \\
\hline DMRT & * & Ns & $*$ & $* * *$ & $* * *$ & $* * *$ & $* * *$ \\
\hline CV (\%) & 12.2 & 29.4 & 23.6 & 14.5 & 15.6 & 26.3 & 9 \\
\hline
\end{tabular}

Ns: nonsignificant at $p<0.05 ;{ }^{*}$ : significant at $p<0.05$; ${ }^{*}$ Significant at $p<0.001$; DAP: days after planting; AUDPC: area under disease progress curve; GM: grand mean; DMRT: Duncan's multiple range test; CV: coefficient of variation.

TABLE 3: The mean of garlic rust disease incidence, PSI at d/t days, and AUDPC data at Segno in 2019 cropping season.

\begin{tabular}{|c|c|c|c|c|c|c|c|}
\hline \multirow[b]{2}{*}{ Trt } & \multirow{2}{*}{$\begin{array}{l}\text { Incidence } \\
\text { 72DAP }\end{array}$} & \multicolumn{5}{|c|}{ PSI } & \multirow{2}{*}{$\begin{array}{l}\text { AUDPC } \\
\% \text { in days }\end{array}$} \\
\hline & & 72DAP & 82DAP & 92DAP & 102DAP & $112 \mathrm{DAP}$ & \\
\hline $0.5 * 1 \mathrm{X}$ & $100^{c}$ & 2 & $18^{\mathrm{a}}$ & $36.67^{\mathrm{d}}$ & $46.67^{\mathrm{d}}$ & $63.33^{\mathrm{e}}$ & $1340^{\mathrm{f}}$ \\
\hline $0.5 * 2 \mathrm{X}$ & $86.67^{\mathrm{abc}}$ & 0 & $18.67^{\mathrm{a}}$ & $36^{\mathrm{d}}$ & $40^{\mathrm{cd}}$ & $35.33^{\mathrm{abc}}$ & $1123^{\mathrm{def}}$ \\
\hline $0.5 * 3 \mathrm{X}$ & $80^{\mathrm{abc}}$ & 2.67 & $18^{\mathrm{a}}$ & $21.33^{\mathrm{ab}}$ & $22^{\mathrm{a}}$ & $21.33^{\mathrm{a}}$ & $733^{\mathrm{a}}$ \\
\hline $0.75 * 1 \mathrm{X}$ & $80^{\mathrm{abc}}$ & 5.33 & $17.33^{\mathrm{a}}$ & $29.33^{\mathrm{abcd}}$ & $42.67^{\mathrm{cd}}$ & $60^{\mathrm{e}}$ & $1220^{\mathrm{def}}$ \\
\hline $0.75 * 2 \mathrm{X}$ & $76.67^{\mathrm{ab}}$ & 2.67 & $15.33^{\mathrm{a}}$ & $33.33^{\mathrm{bcd}}$ & $34.67^{\mathrm{bc}}$ & $41.67^{\mathrm{bc}}$ & $1055^{\text {cde }}$ \\
\hline $0.75 * 3 \mathrm{X}$ & $80^{\mathrm{abc}}$ & 0 & $17.33^{\mathrm{a}}$ & $22.33^{\mathrm{abc}}$ & $27.67^{\mathrm{ab}}$ & $26^{\mathrm{a}}$ & $803^{\mathrm{abc}}$ \\
\hline $1 * 1 \mathrm{X}$ & $100^{c}$ & 4.67 & $18.67^{\mathrm{a}}$ & $28.67^{\mathrm{abcd}}$ & $44.67^{\mathrm{cd}}$ & $59.33^{\mathrm{e}}$ & $1240^{\mathrm{def}}$ \\
\hline $1 * 2 \mathrm{X}$ & $83.33^{\mathrm{abc}}$ & 6 & $17.33^{\mathrm{a}}$ & $32.67^{\mathrm{bcd}}$ & $46^{\mathrm{cd}}$ & $49.33^{\text {cde }}$ & $1237^{\text {def }}$ \\
\hline $1 * 3 \mathrm{X}$ & $90^{\mathrm{abc}}$ & 4 & $17.33^{\mathrm{a}}$ & $19^{\mathrm{a}}$ & $21^{\mathrm{a}}$ & $20.67^{\mathrm{a}}$ & $697^{\mathrm{a}}$ \\
\hline $1.25 * 1 \mathrm{X}$ & $100^{c}$ & 2.67 & $20^{\mathrm{a}}$ & $32^{\mathrm{bcd}}$ & $44.67^{\mathrm{cd}}$ & $60.67^{\mathrm{e}}$ & $1283^{\mathrm{ef}}$ \\
\hline $1.25 * 2 \mathrm{X}$ & $86.67^{\mathrm{abc}}$ & 4.67 & $18^{\mathrm{a}}$ & $28^{\mathrm{abcd}}$ & $43.33^{\mathrm{cd}}$ & $44.67^{\mathrm{cd}}$ & $1140^{\text {def }}$ \\
\hline $1.25 * 3 \mathrm{X}$ & $86.67^{\mathrm{abc}}$ & 3.33 & $18.67^{\mathrm{a}}$ & $26^{\mathrm{abcd}}$ & $28^{\mathrm{ab}}$ & $25.33^{\mathrm{a}}$ & $870^{\mathrm{abc}}$ \\
\hline $1.5 * 1 \mathrm{X}$ & $96.67^{b c}$ & 2.67 & $20^{\mathrm{a}}$ & $33.33^{\mathrm{bcd}}$ & $46^{\mathrm{cd}}$ & $56^{\mathrm{de}}$ & $1287^{\mathrm{ef}}$ \\
\hline $1.5 * 2 \mathrm{X}$ & $70^{\mathrm{a}}$ & 1.33 & $14^{\mathrm{a}}$ & $34.67^{\mathrm{cd}}$ & $36^{\mathrm{bcd}}$ & $28.67^{\mathrm{ab}}$ & $997^{\mathrm{bcd}}$ \\
\hline $1.5 * 3 \mathrm{X}$ & $76.67^{\mathrm{ab}}$ & 2.67 & $15.33^{\mathrm{a}}$ & $25.33^{\mathrm{abcd}}$ & $23.33^{\mathrm{a}}$ & $21.67^{\mathrm{a}}$ & $762^{\mathrm{ab}}$ \\
\hline Control & $100^{c}$ & 10.67 & $30.67^{\mathrm{b}}$ & $50.67^{\mathrm{e}}$ & $59.33^{\mathrm{e}}$ & $78.67^{f}$ & $1853^{g}$ \\
\hline GM & 87.1 & 3.46 & 18.42 & 30.58 & 37.88 & 43.3 & 1102 \\
\hline DMRT & $*$ & $\mathrm{~ns}$ & $*$ & $* * *$ & $* * *$ & $* * *$ & $* * *$ \\
\hline CV (\%) & 13.1 & 96.4 & 21.7 & 21.8 & 16 & 18.1 & 12.7 \\
\hline
\end{tabular}

Ns: non-significant at $p<0.05 ;{ }^{*}$ : significant at $p<0.05 ;{ }^{* *}$ : significant at $p<0.001$; DAP: days after planting; AUDPC: area under disease progress curve; GM: grand mean; DMRT: Duncan's multiple range test; CV: coefficient of variation.

scored from plots treated with 1.5 liters with three times spraying frequencies at Kabe (Table 2) and also 697\%-days scored from 1 liter with three times spray at Segno (Table 3). Among fungicide sprayed plots, the lowest AUDPC was recorded on plots treated with all rates with three times spraying frequencies and the highest was on plots treated with one-time spraying frequencies and control plot. These indicated that spraying fungicide on garlic fields infected with garlic rust at different spraying frequencies has a different effect on garlic rust development. The present study was supported by Tilahun et al.'s [18] research result; maximum AUDPC was scored from unsprayed control plot.

\subsection{Yield and Yield Component Data Assessment}

3.5.1. Bulb Diameter. Tilt spray at different rates and frequencies showed significant difference $(p<0.05)$ in bulb diameter at both locations (Tables 4 and 5). At Kabe, the 
TABLE 4: The mean of garlic bulb yield and yield component data at Kabe in 2019 cropping season.

\begin{tabular}{lcccc}
\hline Treatments & BD $(\mathrm{cm})$ & BW $(\mathrm{g})$ & CW $(\mathrm{g})$ & By $\left(\mathrm{kg} \mathrm{ha}^{-1}\right)$ \\
\hline $0.5 * 1 \mathrm{X}$ & $3.83^{\mathrm{cde}}$ & $29.96^{\mathrm{cd}}$ & $1.78^{\mathrm{cd}}$ & $5209^{\mathrm{cd}}$ \\
$0.5 * 2 \mathrm{X}$ & $4.7^{\mathrm{a}}$ & $37.3^{\mathrm{abcd}}$ & $2.15^{\mathrm{abcd}}$ & $6752^{\mathrm{abc}}$ \\
$0.5 * 3 \mathrm{X}$ & $4.63^{\mathrm{ab}}$ & $45.13^{\mathrm{abc}}$ & $2.52^{\mathrm{a}}$ & $7306^{\mathrm{abc}}$ \\
$0.75 * 1 \mathrm{X}$ & $3.9^{\mathrm{cde}}$ & $32.37^{\mathrm{bcd}}$ & $1.84^{\mathrm{bcd}}$ & $5653^{\mathrm{bcd}}$ \\
$0.75 * 2 \mathrm{X}$ & $4.63^{\mathrm{ab}}$ & $42.83^{\mathrm{abc}}$ & $1.95^{\mathrm{abcd}}$ & $6560^{\mathrm{abcd}}$ \\
$0.75 * 3 \mathrm{X}$ & $4.33^{\mathrm{abcd}}$ & $44.8^{\mathrm{abc}}$ & $2^{\mathrm{abcd}}$ & $7252^{\mathrm{abc}}$ \\
$1 * 1 \mathrm{X}$ & $4^{\mathrm{abcd}}$ & $35.53^{\mathrm{bcd}}$ & $1.89^{\mathrm{abcd}}$ & $5273^{\mathrm{bcd}}$ \\
$1 * 2 \mathrm{X}$ & $4.13^{\mathrm{abcd}}$ & $43.37^{\mathrm{abc}}$ & $2.15^{\mathrm{abcd}}$ & $6257^{\mathrm{abcd}}$ \\
$1 * 3 \mathrm{X}$ & $4.47^{\mathrm{abcd}}$ & $46.17^{\mathrm{ab}}$ & $2.46^{\mathrm{ab}}$ & $7757^{\mathrm{a}}$ \\
$1.25 * 1 \mathrm{X}$ & $3.77^{\mathrm{de}}$ & $39.27^{\mathrm{abcd}}$ & $2.1^{\mathrm{abcd}}$ & $5952^{\mathrm{abcd}}$ \\
$1.25 * 2 \mathrm{X}$ & $4.27^{\mathrm{abcd}}$ & $37.17^{\mathrm{abcd}}$ & $2.21^{\mathrm{abc}}$ & $7377^{\mathrm{ab}}$ \\
$1.25 * 3 \mathrm{X}$ & $4.5^{\mathrm{abc}}$ & $52.8^{\mathrm{a}}$ & $2.49^{\mathrm{a}}$ & $8047^{\mathrm{a}}$ \\
$1.5 * 1 \mathrm{X}$ & $3.95^{\mathrm{bcde}}$ & $36.05^{\mathrm{bcd}}$ & $1.82^{\mathrm{bcd}}$ & $5402^{\mathrm{bcd}}$ \\
$1.5 * 2 \mathrm{X}$ & $4.47^{\mathrm{abcd}}$ & $42.47^{\mathrm{abc}}$ & $1.9^{\mathrm{abcd}}$ & $7317^{\mathrm{abc}}$ \\
$1.5 * 3 \mathrm{X}$ & $4.3^{\mathrm{abcd}}$ & $44.31^{\mathrm{abc}}$ & $2.39^{\mathrm{abc}}$ & $7993^{\mathrm{a}}$ \\
Control & $3.63^{\mathrm{e}}$ & $25.77^{\mathrm{d}}$ & $1.56^{\mathrm{d}}$ & $4660^{\mathrm{d}}$ \\
\hline GM & 4.22 & 39.7 & 2.07 & 6548 \\
DMRT & $*$ & $*$ & $*$ & $* *$ \\
CV $(\%)$ & 8.8 & 20.5 & 15.7 & 16.5 \\
\hline
\end{tabular}

*: significant at $p<0.05 ;{ }^{* *}$ : significant at $p<0.01$;D: bulb diameter; BW: bulb wight; CW: clove weight; BY: bulb yield; GM: grand mean; DMRT: Duncan's multiple range test; CV: coefficient of variation.

TABLE 5: The mean of garlic bulb yield and yield component data at Segno in 2019 cropping season.

\begin{tabular}{lcccc}
\hline Treatment & BD $(\mathrm{cm})$ & BW $(\mathrm{gm})$ & CW $(\mathrm{gm})$ & BY $\left(\mathrm{kg} \mathrm{ha}^{-1}\right)$ \\
\hline $0.5 * 1 \mathrm{X}$ & $3.97^{\mathrm{de}}$ & $30.02^{\mathrm{d}}$ & $1.69 \mathrm{~b}^{\mathrm{c}}$ & $4954^{\mathrm{c}}$ \\
$0.5 * 2 \mathrm{X}$ & $4^{\mathrm{d}}$ & $34.26^{\mathrm{cd}}$ & $1.86^{\mathrm{abc}}$ & $6496^{\mathrm{abc}}$ \\
$0.5 * 3 \mathrm{X}$ & $4.8^{\mathrm{ab}}$ & $58.28^{\mathrm{ab}}$ & $2.74^{\mathrm{ab}}$ & $7272^{\mathrm{abc}}$ \\
$0.75 * 1 \mathrm{X}$ & $4.07^{\mathrm{cd}}$ & $33.38^{\mathrm{cd}}$ & $1.8^{\mathrm{abc}}$ & $5284^{\mathrm{bc}}$ \\
$0.75 * 2 \mathrm{X}$ & $4.43^{\mathrm{abc}}$ & $48.82^{\mathrm{abcd}}$ & $2.4^{\mathrm{abc}}$ & $6552^{\mathrm{abc}}$ \\
$0.75 * 3 \mathrm{X}$ & $4.83^{\mathrm{a}}$ & $54.57^{\mathrm{abc}}$ & $2.86^{\mathrm{a}}$ & $7784^{\mathrm{ab}}$ \\
$1 * 1 \mathrm{X}$ & $4.6^{\mathrm{ab}}$ & $34.85^{\mathrm{bcd}}$ & $1.59^{\mathrm{c}}$ & $5435^{\mathrm{bc}}$ \\
$1 * 2 \mathrm{X}$ & $4.87^{\mathrm{a}}$ & $46.47^{\mathrm{abcd}}$ & $2.46^{\mathrm{abc}}$ & $6365^{\mathrm{abc}}$ \\
$1 * 3 \mathrm{X}$ & $4.63^{\mathrm{ab}}$ & $59.34^{\mathrm{a}}$ & $2.84^{\mathrm{a}}$ & $7692^{\mathrm{ab}}$ \\
$1.25 * 1 \mathrm{X}$ & $4.7^{\mathrm{ab}}$ & $40.4^{\mathrm{abcd}}$ & $2.16^{\mathrm{abc}}$ & $5509^{\mathrm{bc}}$ \\
$1.25 * 2 \mathrm{X}$ & $4.37^{\mathrm{bcd}}$ & $44.58^{\mathrm{abcd}}$ & $2.2^{\mathrm{abc}}$ & $6814^{\mathrm{abc}}$ \\
$1.25 * 3 \mathrm{X}$ & $4.7^{\mathrm{ab}}$ & $54.89^{\mathrm{abc}}$ & $2.7^{\mathrm{ab}}$ & $7755^{\mathrm{ab}}$ \\
$1.5 * 1 \mathrm{X}$ & $3.93^{\mathrm{de}}$ & $47.8^{\mathrm{abcd}}$ & $2.29^{\mathrm{abc}}$ & $6121^{\mathrm{abc}}$ \\
$1.5 * 2 \mathrm{X}$ & $4.6^{\mathrm{ab}}$ & $40.95^{\mathrm{abcd}}$ & $2.11^{\mathrm{abc}}$ & $6290^{\mathrm{abc}}$ \\
$1.5 * 3 \mathrm{X}$ & $4.86^{\mathrm{ab}}$ & $58.36^{\mathrm{ab}}$ & $2.82^{\mathrm{a}}$ & $8088^{\mathrm{a}}$ \\
Control & $3.57^{\mathrm{e}}$ & $26.25^{\mathrm{d}}$ & $1.55^{\mathrm{c}}$ & $4740^{\mathrm{c}}$ \\
\hline GM & 4.43 & 44.58 & 2.25 & 6446.85 \\
DMRT & $* * *$ & $*^{\mathrm{a}}$ & $*$ & $*$ \\
CV $(\%)$ & 5.3 & 27.2 & 24.3 & 20.2 \\
\hline
\end{tabular}

*: significant at $p<0.05{ }^{* * *}$ : significant at $p<0.001$; BD: bulb diameter; BW: bulb wight; CW: clove weight; BY: bulb yield; GM: grand mean; DMRT: Duncan's multiple range test; CV: coefficient of variation.

highest $(4.7 \mathrm{~cm})$ bulb diameter was recorded from plots treated with 0.5 liters with two times spray frequencies, whereas the lowest $(3.63 \mathrm{~cm})$ bulb diameter was recorded from an unsprayed control plot (Table 4). Similarly, at Segno the highest $(4.86 \mathrm{~cm})$ bulb diameter was recorded from plots treated with 0.5 liters with two times spray frequencies whereas the lowest $(3.57 \mathrm{~cm})$ bulb diameter was recorded from unsprayed control plot (Table 5). The present study is in line with Habtewold et al. [10]; the highest bulb diameter $(3.17 \mathrm{~cm})$ was recorded on bulbs from Tilt 250EC sprayed plot while the smallest $(2.65 \mathrm{~cm})$ was from unsprayed plots.

3.5.2. Bulb Weight. The analysis of variance showed a significant difference at $p<0.05$ between the combination of rate and spraying frequencies of Tilt application on bulb weights (Tables 4 and 5). At Kabe, from all combinations of these rate and spraying frequencies of Tilt fungicide, the maximum $(52.8 \mathrm{~g})$ bulb weight was recorded on plots treated with $1.5 \mathrm{~L}$ propiconazole with three times spraying of propiconazole. On the other hand, the minimum $(25.77 \mathrm{~g})$ garlic weight was obtained from the untreated control plot (Table 4). Similarly, at Segno the maximum (59.34g) and minimum $(26.25 \mathrm{~g})$ bulb weight were recorded on plots sprayed with $1 \mathrm{~L}$ with three times spraying frequency of propiconazole and from untreated control plots, respectively (Table 5). Similarly, the average weight of bulbs harvested from fungicide sprayed plots was significantly $(p<0.05)$ higher than the weight of bulbs that were harvested from the unsprayed plots [13]. All the fungicide-treated plots gave higher weight than the unsprayed plots [8].

3.5.3. Clove Weight. The result of average clove weight revealed a significant difference $(p<0.05)$ among the combination of rate and spraying frequencies of Tilt fungicide at both locations of Kabe and Segno (Tables 4 and 5). At Kabe, the highest average clove weight $(2.49 \mathrm{~g})$ was recorded from plots treated with $1.25 \mathrm{~L}$ propiconazole with three times spraying frequency whereas the lowest clove weight $(1.56 \mathrm{~g})$ was recorded from untreated control plots (Table 4). Similarly, at Segno the highest (2.86 g) and lowest $(1.55 \mathrm{~g})$ average clove weight were obtained on plots sprayed with $0.75 \mathrm{~L}$ with three times spraying frequency of propiconazole and from untreated control plots, respectively (Table 5). It is similar to the result of Worku and Dejene's [9] research findings; all fungicide-treated plots gave the highest clove weight compared to unsprayed control plots.

3.5.4. Bulb Yield. A significant difference $(p<0.05)$ among treatments was recorded for bulb yield at both locations (Tables 4 and 5). The highest $\left(8047 \mathrm{~kg} \mathrm{ha}^{-1}\right)$ bulb yield was obtained from plots that were treated with $1.25 \mathrm{~L}$ with three times spraying frequency of propiconazole and the lowest $\left(4660 \mathrm{~kg} \mathrm{ha}^{-1}\right)$ found from untreated control plots at Kabe (Table 4). At Segno, the highest $\left(8088 \mathrm{~kg} \mathrm{ha}^{-1}\right.$ ) bulb yield was also obtained from $1.5 \mathrm{~L}$ with three times spraying frequency of propiconazole and the lowest $\left(4740 \mathrm{~kg} \mathrm{ha}^{-1}\right)$ found from untreated control plots (Table 5). Plots sprayed with the fungicide three times at each fungicide rate had given maximum yield compared to two and one times spraying frequencies at both locations. It indicates spraying frequency is the more determining factor than different rates of propiconazole for the control of garlic rust. The result agrees with Admasie et al.'s [11] research result; the highest total bulb yield was obtained from plots that were sprayed with propiconazole fungicide. Habtewold et al. [11] also revealed 
that the highest bulb yield was obtained from Rex Duo and propiconazole fungicides. In addition to that, the highest bulb yield was obtained from propiconazole sprayed plots as compared with unsprayed plots [18].

3.6. Relative Yield Loss and Percentage Yield Increase. Relative yield loss and percentage yield advantage were calculated by using the average bulb yield of Kabe and Segno locations. Because both Kabe and Segno locations are found under Woreilu District, the information of relative yield loss and percentage yield advantage was calculated as a district. The yield loss assessment was calculated for all treatments relative to the yield of a maximum protected plot of 1.5 fungicide rate with three times spraying frequency of propiconazole applications. The highest $(41.55 \%)$ relative yield loss was recorded on unsprayed control plots, followed by $36.8 \%$ yield loss from plots treated with $1 \mathrm{~L}$ with one time spraying frequency of propiconazole application (Table 6). The combinations of rate and spraying frequencies of propiconazole applications relatively reduced garlic bulb yield losses as compared with the unsprayed control plot. This indicates how much garlic rust disease is damaging garlic plants during favorable conditions when effective management practices have not been undertaken. The results indicated the application of fungicides used to minimize the effect of the disease and decreased bulb yield losses. According to Negash et al. [19], all fungicide-treated plots reduced bulb yield losses of garlic as compared with control plots.

The percent yield increase study was calculated from all treatments as compared to the untreated control plots. The present study indicated that the percent yield increase showed differences among treatments. The highest (71.07\%) yield increase was obtained from plots treated with $1.5 \mathrm{~L}$ fungicide rate with three times spraying frequency of propiconazole applications, followed by $68.11 \%$ yield increase from plots treated with $1.25 \mathrm{~L}$ with three times spraying frequency of tilt fungicide application (Table 6). The present study is supported by Habtewold et al.'s [10] research findings; $55.9 \%$ yield advantage was obtained from propiconazole sprayed plot.

3.7. Correlation Coefficients of Yield, Yield Components, and Disease Parameters. The associations of yield, yield component, and disease parameters were evaluated using correlation analysis. The disease parameters, namely, the final percentage severity index (112DAP) and AUDPC, were highly significantly $(p<0.01)$ correlated with each other (Table 7). Correlation coefficients between the percentage severity index at final disease assessment and AUDPC were strongly correlated $\left(r=0.967^{* *}\right.$ and $\left.r=0.949^{* *}\right)$ at Kabe and Segno, respectively. The correlation coefficient of bulb yield with all disease parameters was significant $(p<0.01)$ and negatively correlated $\left(r=-0.824^{* *}\right.$ and $\left.-0.722^{* *}\right)$ with the final percentage severity index (112DAP) and AUDPC, respectively, at Kabe. Also, at Segno bulb yield was negatively correlated $\left(r=-0.929^{* *}\right.$ and $\left.-0.886^{* *}\right)$ with percentage severity index and AUDPC. Moreover, bulb weight $\left(r=0.858^{* * *}\right.$ and $\left.0.918^{* * *}\right)$ and clove weight $\left(r=0.841^{* * *}\right.$ and $0.916^{* * *}$ ) were positively correlated with bulb yield at Kabe and Segno, respectively. Yield and yield components were negatively correlated with percentage severity index and AUDPC (Table 7). Similarly, the present study supported the research findings of [19]; correlation analysis revealed significant $(p<0.001)$ negative relationships between garlic rust severity and total yield, and clove weight.

3.8. Linear Regression between AUDPC and Total Bulb Yield. Linear regression of the AUDPC was used for predicting the garlic bulb yield loss, because AUDPC linear regression is a better analytical model to indicate the relationship of yield loss with the disease effects. AUDPC was considered to be an independent variable and total bulb yield considered as a dependent variable was regressed to estimate the yield loss due to the disease. Thus, as AUDPC increases, the yield decreases and goes towards zero asymptotes, which indicates the inverse relation between AUDPC and bulb yield of garlic.

At Kabe, equation of the model was $Y=-2.8059 X+9395.6$ which indicated that, for every one unit increase in AUDPC (\% days), there was a corresponding $2.8059 \mathrm{~kg} \mathrm{ha}^{-1}$ garlic yield loss that occurred during 2019 main cropping season (Figure 1). Regarding the relationship indicated by regression linear model, $52.18 \%$ of loss in garlic yield was predicted due to garlic rust (Puccinia allii). Similarly, the equation of linear regression model was $Y=-3.1979 X+9972.6$ which indicated that, for every one unit increase in AUDPC (\% days), there was a corresponding $3.1979 \mathrm{~kg} \mathrm{ha}^{-1}$ garlic yield loss that occurred during Segno 2019 main cropping season. The model indicated that $78.48 \%$ of loss in garlic yield was predicted due to garlic rust disease at Segno. In general, the linear regression graph indicates that as the AUDPC increased, there was a decreasing trend in garlic bulb yield at both locations.

3.9. Cost-Benefit Analysis. Cost-benefit analysis was done for the management of garlic rust disease through different rates and spraying frequencies of propiconazole application (Table 8). The result of partial budget analysis of Tilt fungicide application on garlic field showed an increase in net benefit due to fungicide application which resulted in higher total bulb yield than unsprayed plots. Three times spraying frequencies were giving the highest net benefit, marginal net benefit, and marginal rate of return on all rates as compared to other rates and unsprayed treatments. Maximum (489698 ETB $\mathrm{ha}^{-1}$ ) and minimum (255452 ETB ha ${ }^{-1}$ ) net benefits were obtained from plots treated with $1.5 \mathrm{~L}$ with three times spraying and unsprayed plots, respectively. Marginal net benefit had shown variations among the combination of rate and spraying frequency of propiconazole. The maximum $\left(234246 \mathrm{ETB} \mathrm{ha}^{-1}\right)$ and minimum (26478 ETB ha-1) marginal net benefit were recorded from $1.5 \mathrm{~L}$ with three times spraying and $0.5 \mathrm{~L}$ with one time spraying, respectively. In addition to that, marginal rate of return was described as a method for comparing the costs that vary with the net benefits of all treatments. The highest (6896.36\%) marginal 
TABLE 6: Relative yield loss and percentage yield increase for both locations during 2019 cropping season.

\begin{tabular}{lccc}
\hline Treatment & BY $\left(\mathrm{kg} \mathrm{ha}^{-1}\right)$ & RYL $(\%)$ & PYI $(\%)$ \\
\hline $0.5 * 1 \mathrm{X}$ & 5081.5 & 36.80 & 8.12 \\
$0.5 * 2 \mathrm{X}$ & 6624 & 17.62 & 40.94 \\
$0.5 * 3 \mathrm{X}$ & 7289 & 9.35 & 55.09 \\
$0.75 * 1 \mathrm{X}$ & 5468.5 & 31.99 & 16.35 \\
$0.75 * 2 \mathrm{X}$ & 6556 & 6.46 & 39.49 \\
$0.75 * 3 \mathrm{X}$ & 7518 & 33.41 & 59.96 \\
$1 * 1 \mathrm{X}$ & 5354 & 21.51 & 3.91 \\
$1 * 2 \mathrm{X}$ & 6311 & 3.93 & \\
$1 * 3 \mathrm{X}$ & 7724.5 & 28.73 & \\
$1.25 * 1 \mathrm{X}$ & 5730.5 & 11.75 & \\
$1.25 * 2 \mathrm{X}$ & 7095.5 & 1.73 & 21.93 \\
$1.25 * 3 \mathrm{X}$ & 7901 & 28.34 & 50.97 \\
$1.5 * 1 \mathrm{X}$ & 5761.5 & 15.38 & 68.11 \\
$1.5 * 2 \mathrm{X}$ & 6803.5 & 0.00 & 22.59 \\
$1.5 * 3 \mathrm{X}$ & 8040.5 & 41.55 & 74.76 \\
Control & 4700 & 0.07 \\
\hline
\end{tabular}

BY: bulb yield; RYL: relative yield loss; PYI: percentage yield increase.

TABLE 7: Correlation coefficients of bulb yield, yield component, and disease parameters at Kabe and Segno during 2019 cropping season.

\begin{tabular}{lcccccccccc}
\hline & \multicolumn{3}{c}{ Kabe } & \multicolumn{3}{c}{ Segno } \\
& PSI & AUDPC & BW & CW & By & PSI & AUDPC & BW & CW & BY \\
\hline PSI & 1 & - & - & - & - & 1 & - & - & - \\
AUDPC & $0.967^{* *}$ & 1 & - & - & - & $0.949^{* *}$ & 1 & - & - \\
BW & $-0.827^{* *}$ & $-0.744^{* *}$ & 1 & - & - & $-0.832^{* *}$ & $-0.857^{* *}$ & 1 & - \\
CW & $-0.706^{* *}$ & $-0.632^{* *}$ & $0.813^{* *}$ & 1 & - & $-0.821^{* *}$ & $-0.825^{* *}$ & $0.975^{* *}$ & 1 \\
BY & $-0.824^{* *}$ & $-0.722^{* *}$ & $0.858^{* *}$ & $0.841^{* *}$ & 1 & $-0.929^{* *}$ & $-0.886^{* *}$ & $0.918^{* *}$ & $0.916^{* *}$ & 1 \\
\hline
\end{tabular}

${ }^{* *}$ : correlation is significant at the 0.01 level; $*$ : correlation is significant at the 0.05 level; PSI: percentage severity index; AUDPC: area under disease progress curve; BW: bulb weight; CW: clove weight; BY: bulb yield.
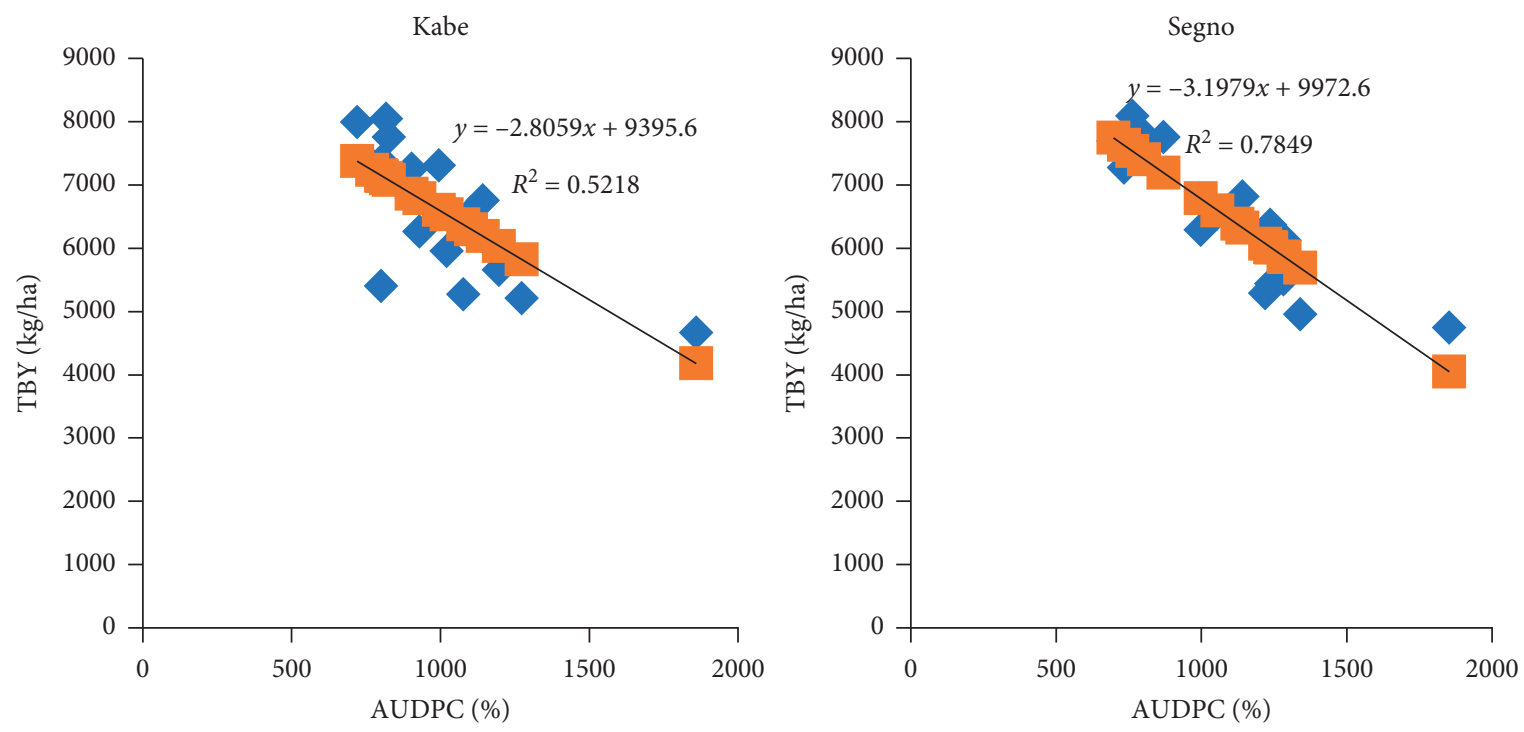

Figure 1: Linear regressions of garlic bulb yield and AUDPC at Kabe and Segno during the 2019 main cropping season. 
TABLE 8: Partial budget analysis of rate and spraying frequencies of propiconazole for the mgt of garlic rust.

\begin{tabular}{|c|c|c|c|c|c|c|c|c|c|}
\hline TRT & BY $(\mathrm{kg} / \mathrm{ha})$ & $\mathrm{ABY}$ & $\mathrm{PP} / \mathrm{Kg}$ & GB/ha & TIC & $\mathrm{MC}$ & $\mathrm{NB}$ & MNB & MRR \\
\hline $0.5 * 1 \mathrm{X}$ & 5081.5 & 4573.4 & 80 & 365868 & 83938 & 990 & 281930 & 26478 & 2674.55 \\
\hline $0.5 * 2 \mathrm{X}$ & 6624 & 5961.6 & 80 & 476928 & 84928 & 1980 & 392000 & 136548 & 6896.36 \\
\hline $0.5 * 3 \mathrm{X}$ & 7289 & 6560.1 & 80 & 524808 & 85918 & 2970 & 438890 & 183438 & 6176.36 \\
\hline $0.75 * 1 \mathrm{X}$ & 5468.5 & 4921.7 & 80 & 393732 & 84213 & 1265 & 309519 & 54067 & 4274.07 \\
\hline $0.75 * 2 \mathrm{X}$ & 6556 & 5900.4 & 80 & 472032 & 85478 & 2530 & 386554 & 131102 & 5181.90 \\
\hline $0.75 * 3 \mathrm{X}$ & 7518 & 6766.2 & 80 & 541296 & 86743 & 3795 & 454553 & 199101 & 5246.40 \\
\hline $1 * 1 \mathrm{X}$ & 5354 & 4818.6 & 80 & 385488 & 84488 & 1540 & 301000 & 45548 & 2957.66 \\
\hline $1 * 2 \mathrm{X}$ & 6311 & 5679.9 & 80 & 454392 & 86028 & 3080 & 368364 & 112912 & 3665.97 \\
\hline $1 * 3 \mathrm{X}$ & 7724.5 & 6952.1 & 80 & 556164 & 87568 & 4620 & 468596 & 213144 & 4613.51 \\
\hline $1.25 * 1 \mathrm{X}$ & 5730.5 & 5157.5 & 80 & 412596 & 84763 & 1815 & 327833 & 72381 & 3987.93 \\
\hline $1.25 * 2 \mathrm{X}$ & 7095.5 & 6386.0 & 80 & 510876 & 86578 & 3630 & 424298 & 168846 & 4651.40 \\
\hline $1.25 * 3 \mathrm{X}$ & 7901 & 7110.9 & 80 & 568872 & 88393 & 5445 & 480479 & 225027 & 4132.73 \\
\hline $1.5 * 1 \mathrm{X}$ & 5761.5 & 5185.4 & 80 & 414828 & 85038 & 2090 & 329790 & 74338 & 3556.84 \\
\hline $1.5 * 2 \mathrm{X}$ & 6803.5 & 6123.2 & 80 & 489852 & 87128 & 4180 & 402724 & 147272 & 3523.25 \\
\hline $1.5 * 3 \mathrm{X}$ & 8040.5 & 7236.5 & 80 & 578916 & 89218 & 6270 & 489698 & 234246 & 3735.98 \\
\hline Control & 4700 & 4230.0 & 80 & 338400 & 82948 & 0 & 255452 & 0 & 0.00 \\
\hline
\end{tabular}

BY: bulb yield; ABY: adjusted bulb yield; PP: price per kg; GB: gross benefit; TIC: total input cost; MC: marginal cost; NB: net benefit; MNB: marginal net benefit; MRR: marginal rate of return.

rate of return had been obtained from plots treated with $0.5 \mathrm{~L}$ with two times spraying, followed by $6176.36 \%$ obtained from $0.5 \mathrm{~L}$ with three times spraying frequency of propiconazole. The result agrees with Yonas' [13] research result; the highest $(134,830$ ETB) net benefit was obtained from spraying of Nativo twice at 21-day interval starting from the onset of the disease.

\section{Conclusion and Recommendation}

The present study was carried out to determine an appropriate rate and spray frequency of propiconazole for the management of garlic rust and select cost-effective management options of the disease at two farms (Kabe and Segno Kebele) of Woreilu District during 2019 main cropping season. The combination of rate and spraying frequency of Tilt fungicide showed a significant difference in percentage severity index, area under disease progress curve (AUDPC), yield, and yield components. The combination of two and three times spraying frequency with all rates of propiconazole fungicide reduced disease severity with corresponding increase in bulb yield. The highest (79.33 and $78.67 \%$ ) PSI at 112DAP was scored from unsprayed control plots at Kabe and Segno, respectively. Also, the lowest $(17.33 \%)$ was scored from plots treated with $1.5 \mathrm{~L}$ with three times spray at Kabe, and at Segno the lowest (20.67\%) was scored from $1 \mathrm{~L}$ with three times spraying frequency.

The highest $\left(8047 \mathrm{~kg} \mathrm{ha}^{-1}\right.$ ) yield was obtained from plots that were treated with $1.25 \mathrm{~L}$ with three times spraying frequency at Kabe and $8047 \mathrm{~kg} \mathrm{ha}^{-1}$ yield was also obtained from $1.5 \mathrm{~L}$ with three times spraying frequency of propiconazole at Segno and the lowest (4660 and $4740 \mathrm{~kg} \mathrm{ha}^{-1}$ ) was found from untreated control plots at Kabe and Segno, respectively. Plots sprayed with the fungicide three times at each fungicide rate had given maximum yield compared to two and one time spraying frequencies at both locations. It indicates spraying frequency is the more determining factor than different rates of propiconazole for the control of garlic rust disease. The partial budget analysis proved that the highest (6896.36\%) marginal rate of return had been obtained from plots treated with $0.5 \mathrm{~L}$ with two times spraying, followed by $6176.36 \%$ obtained from $0.5 \mathrm{~L}$ with three times spraying frequency of propiconazole fungicide.

Generally, from this finding, the use of $0.5 \mathrm{~L}$ propiconazole with two times spraying frequency is recommended for end-users, because it gave relatively the highest economic benefit as compared to the other treatments and the control. However, the experiment was done for one year, so it needs further study under different agro-ecologies and additional years to determine the best-integrated management options and to increase the production and productivity of garlic in the country.

\section{Data Availability}

The data used to support the findings of this study are available from the corresponding author upon request.

\section{Conflicts of Interest}

There are no conflicts of interest related to the publication of this research manuscript.

\section{Acknowledgments}

The authors would like to thank Sirinka Agricultural Research Center and Amhara Agricultural Research Institute for their full budget support.

\section{References}

[1] Allen, J. (2009). Garlic Production. Factsheet, Garlic Production, Order Number 97-007. http://www.omafra.gov.on. $\mathrm{ca} /$ english/crops/facts/09-011w.htm.

[2] H. D. Tindal, Vegetable in the Tropics, Macmillan Education Limited, Houdmills, EN, UK, 1986. 
[3] R. Kamenetsky and H. D. Rabinowitch, "Floral development in bolting garlic," Sexual Plant Reproduction, vol. 13, no. 4, pp. 235-241, 2001.

[4] C. Voigt, "Glorious garlic herb of the year 2004," Journal of International Herb, pp. 1-6, Association Horticulture Committee, Virginia State University, Petersburg, VA, USA, 2004.

[5] M. J. Kilgori, M. D. Magaji, and A. I. Yakubu, "Productivity of two garlic (Allium sativum L.) cultivars as affected by different levels of nitrogenous and phosphorus fertilizers in sokota, Nigeria," American-eurasian Journal of Agricultural \& Environmental Sciences, vol. 2, no. 2, pp. 158-162, 2007.

[6] A. Kassaw, M. Abera, and E. Belete, "The response of potato late blight to potato varieties and fungicide spraying frequencies at meket, Ethiopia," Cogent Food \& Agriculture, vol. 7, no. 1, Article ID 1870309, 2021.

[7] G. N. Agrios, Plant Pathologyp. 922, 5th edition, Academic Press, San Diego, CA, USA, 2005.

[8] S. T. Koike, R. F. Smith, R. M. Davis, J. J. Nunez, and R. E. Voss, "Characterization and control of garlic rust in California," Plant Disease, vol. 85, no. 6, pp. 585-591, 2001.

[9] Y. Worku and M. Dejene, "Effects of garlic rust (Puccinia allii) on yield and yield components of garlic in bale highlands, south eastern Ethiopia," Journal of Plant Pathology and Microbiology, vol. 3, p. 118, 2012.

[10] H. Kifelew, Z. Dagne, S. Tilahun et al., "Result of fungicides efficacy evaluation for the control of garlic (allium sativum L.) rust (puccinia allii rudolphi) at holetta, Ethiopia," International Journal Of Research Studies In Agricultural Sciences (IJRSAS), vol. 5, no. 5, pp. 1-4, 2019.

[11] A. Kassaw, Abebe Ayalew, and A. Mihrete, "Evaluation of fungicides for the management of garlic rust (Puccinia allii) in Legambo, Eastern Amhara," Alemayehu Assefa and Mulugeta Alemayehu, Amhara Agricultural Research Institute, in Proceeding of the 9th and 10th Annual Regional Conference on Completed Crop Research Activities, March 2017.

[12] M. Worku, T. Azene, and D. Mashilla, "Evaluation of fungicides on the control of garlic rust (puccinia alli) in Eastern Ethiopia," International Journal of Emerging Technology and Advanced Engineering, vol. 6, no. 1, 2016.

[13] Yonas Worku Mulat, "Determination of optimum Nativo SC 300 (trifloxystrobin $100 \mathrm{~g} / \mathrm{l}+$ tebuconazole $200 \mathrm{~g} / \mathrm{l}$ ) spray frequency for control of rust (Puccinia allii rudolphi) on garlic in bale highlands, south eastern Ethiopia," American Journal of Agriculture and Forestry, vol. 5, no. 2, pp. 16-19, 2017.

[14] J. B. J. Wheeler, An Introduction to Plant Diseases, p. 347, Wiley, London, EN, UK, 1969.

[15] C. L. Campbell and L. V. Madden, Introduction to Plant Disease Epidemiology, p. 532, John Wiley \& Sons, New York, NY, USA, 1990.

[16] K. Robert and N. Janes, Seed Pathology, Vol. 2, The Mac Millan Press Ltd, London, EN, UK, 1991.

[17] Lung'aho C., Nyongesa M. and Wakahiu M. (2003). Evaluation of globe ( $6 \%$ cymoxanil/ $70 \%$ mancozeb) for potato late blight management. At http://www.kari.org/fileadmin/ publications/10thproceedings/Volone/EvaluationGlobe.pdf/ 05-10-2018.

[18] T. Negash, H. Shifa, and T. Regassa, "Management of garlic rust (Puccinia allii) through fungicide at bale highlands, south eastern Ethiopia," Food Science and Quality Management, vol. 81, 2018.

[19] T. Negash, H. Shifa, and T. Regassa, "Fungicidal management of garlic rust (Puccinia allii) and assessment of yield losses due to the disease," Journal of Plant Pathology and Microbiology, vol. 10, p. 471, 2019. 